OPEN ACCESS

Edited by: Filippo Brighina, University of Palermo, Italy

Reviewed by: Arun Singh,

The University of lowa, United States

Carmelo Chisari,

University Hospital Pisana, Italy

*Correspondence:

Fioravante Capone f.capone@unicampus.it

Specialty section: This article was submitted to Brain Imaging and Stimulation, a section of the journal Frontiers in Human Neuroscience

Received: 01 October 2019 Accepted: 14 January 2020

Published: 05 February 2020

Citation:

Capone F, Pellegrino $G$, Motolese F, Rossi M, Musumeci G and Di Lazzaro V (2020) Extremely Low Frequency Magnetic Fields Do Not Affect LTP-Like Plasticity in Healthy Humans.

Front. Hum. Neurosci. 14:14. doi: 10.3389/fnhum.2020.00014

\section{Extremely Low Frequency Magnetic Fields Do Not Affect LTP-Like Plasticity in Healthy Humans}

\author{
Fioravante Capone $e^{1,2 *}$, Giovanni Pellegrino ${ }^{3}$, Francesco Motolese ${ }^{1,2}$, \\ Mariagrazia Rossi ${ }^{1,2}$, Gabriella Musumeci ${ }^{1,2}$ and Vincenzo Di Lazzaro ${ }^{1}$ \\ ${ }^{1}$ Unit of Neurology, Neurophysiology, and Neurobiology, Department of Medicine, Università Campus Bio-Medico di Roma, \\ Rome, Italy, ${ }^{2}$ NeXT: Neurophysiology and Neuroengineering of Human-Technology Interaction Research Unit, Campus \\ Bio-Medico University, Rome, Italy, ${ }^{3}$ Neurology and Neurosurgery, Montreal Neurological Institute and Hospital, McGill \\ University, Montreal, QC, Canada
}

Introduction: Several studies explored the biological effects of extremely low-frequency magnetic fields (ELF-MFs) in vitro, reporting the induction of functional changes in neuronal activity. In particular, ELF-MFs can influence synaptic plasticity both in vitro and in animal models but some studies reported an increase in long-term potentiation (LTP) whereas others suggested its reduction. However, no specific study has investigated such effect on humans.

Aims: To evaluate whether ELF-MFs affect the propensity of the human cortex to undergo LTP-like plasticity.

Methods: We designed a randomized, single-blind, sham-controlled, cross-over study on 10 healthy subjects. Cortical plasticity was induced by intermittent theta burst stimulation (iTBS) before and after 45-min ELF-MFs ( $75 \mathrm{~Hz} ; 1.8 \mathrm{mT}$ ) or sham exposure and was estimated by measuring the changes of motor evoked potentials (MEP) amplitude before and after each iTBS.

Results: No adverse events were reported. No significant effects of ELF-MFs on cortical plasticity were found.

Conclusion: Whole-brain exposure to ELF-MFs $(75 \mathrm{~Hz} ; 1.8 \mathrm{mT})$ is safe and does not seem to significantly affect LTP-like plasticity in human motor cortex.

Keywords: magnetic fields, brain stimulation, plasticity, long-term potentiation, low frequency, extremely lowfrequency magnetic fields

\section{INTRODUCTION}

Several studies explored the biological effects of extremely low-frequency $(0-300 \mathrm{~Hz})$ magnetic fields (ELF-MFs) in vitro, reporting the induction of functional changes in neuronal activity (Di Lazzaro et al., 2013). On human subjects, ELF-MFs can produce measurable changes in brain electrical activity and can also influence cerebral functions such as motor control, sensory perception, cognitive activities, sleep, and mood (Bagheri Hosseinabadi et al., 2019). More recently, the potential application of ELF-MFs for non-invasively modulating brain activity has 
been investigated in different neuropsychiatric diseases such as stroke (Capone et al., 2009) and depression (Bagheri Hosseinabadi et al., 2019).

According to the most part of the studies, ELF-MFs are safe and well tolerated (Di Lazzaro et al., 2013). However, previous papers have showed deterioration in memory and learning processes after ELF-MFs exposure both in animals (Jadidi et al., 2007) and in humans (Podd et al., 2002; Corbacio et al., 2011). Instead, other studies have reported, after chronic ELF-MFs exposure, a positive effect in social recognition memory (Varró et al., 2009) and spatial learning (Liu et al., 2015).

Long-term potentiation (LTP) is a form of synaptic plasticity (Bear and Malenka, 1994) and is considered one of the most important molecular mechanisms underlying learning and memory. ELF-MFs affect synaptic plasticity both in vitro (Ahmed and Wieraszko, 2008; Varró et al., 2009; Balassa et al., 2013) and in animal models (Komaki et al., 2014), but type and significance of such effect remain unclear. Indeed, some studies reported an increase of LTP (Ahmed and Wieraszko, 2008; Komaki et al., 2014) whereas others suggested its reduction (Balassa et al., 2013). Moreover, no specific study has investigated such effect in humans.

Recently, protocols of repetitive transcranial magnetic stimulation (rTMS) of the brain resembling experimental LTP models have been introduced. The rTMS paradigm known as intermittent theta burst stimulation (iTBS) produces a prolonged increase of cortical excitability (Huang et al., 2005). The effects of iTBS are influenced by drugs that act at the N-methyl-D-aspartic acid (NMDA) receptor level (Huang et al., 2007), supporting the hypothesis that the after-effects of iTBS involve LTP-like changes. Thus, by this technique, it is possible to evaluate, non-invasively, synaptic plasticity in humans.

Aim of the present study was to evaluate, by means of iTBS, whether ELF-MFs exposure affects the propensity of the cortex to undergo LTP-like plasticity.

\section{MATERIALS AND METHODS}

We designed a randomized, single-blind, sham-controlled, crossover study on 10 healthy subjects ( 7 F, $25 \pm 2$ years). The study was performed according to the Declaration of Helsinki, was approved by the Local Ethics Committee and all participants signed a written informed consent.

\section{Study Design}

Figure 1 graphically depicts the experimental design. Cortical plasticity was estimated by measuring the changes of cortical excitability induced by iTBS before and after $45 \mathrm{~min}$ of exposure to ELF-MFs or to sham stimulation. All the subjects underwent two sessions of the study (REAL or SHAM) at a distance of at least 1 week. The order of the session was counterbalanced between subjects.

\section{Evaluation of Cortical Plasticity}

For the evaluation of cortical excitability, magnetic stimulation was performed with a high-power Magstim 200 (Magstim Co.,
Whitland, Dyfed, United Kingdom). A figure-of-eight coil, with external loop diameter of $9 \mathrm{~cm}$, was held over the motor cortex at the optimum scalp position to elicit motor evoked potentials (MEPs) in the contralateral first dorsal interosseous muscle (FDI). The induced current flowed in a postero-anterior direction. MEPs were recorded via two 9-mm-diameter $\mathrm{Ag}-\mathrm{AgCl}$ surface electrodes with the active electrode over the motor point of the FDI and the reference on the metacarpophalangeal joint of the index finger. The EMG was amplified and filtered (bandwidth $3 \mathrm{~Hz}-3 \mathrm{kHz}$ ) by D360 amplifiers (Digitimer, Welwyn Garden City, Herts, United Kingdom). Data were collected on a computer with a sampling rate of $10 \mathrm{kHz}$ per channel and stored for later analysis using a CED $1401 \mathrm{~A} / \mathrm{D}$ converter (Cambridge Electronic Design, Cambridge, United Kingdom).

At baseline, we measured motor thresholds and MEP amplitude bilaterally. Resting motor threshold (RMT) was defined as the minimum stimulus intensity that produced a liminal MEP (about $50 \mu \mathrm{V}$ in $50 \%$ of ten trials) at rest. Active motor threshold (AMT) was defined as the minimum stimulus intensity that produced a liminal MEP (about $200 \mu \mathrm{V}$ in $50 \%$ of ten trials) during isometric contraction of the tested muscle. A constant level of voluntary contraction was maintained with reference to an oscilloscope display of the EMG signal in front of the subject. Auditory feedback of the EMG activity was also provided. RMT and AMT are given in percentage of maximum stimulator output (\% MSO). For MEP amplitude, the responses to 15 stimuli at an intensity of $120 \%$ RMT were averaged at rest (Di Lazzaro et al., 2008, 2011). Trials contaminated by EMG activity were discarded.

Intermittent theta burst stimulation was delivered over the right motor cortex "hotspot" for MEPs in the contralateral FDI muscle using a DUOMAG XT stimulator (DEYMED Diagnostic, Czech Republic) and a figure-of-eight shaped coil, with the handle pointed posteriorly and approximately perpendicular to the central sulcus. The initial direction of the current induced in the brain was anterior to posterior. The magnetic stimulus had a biphasic waveform with a pulse width of about $280 \mu \mathrm{s}$ and maximum magnetic field strength of $1.5 \mathrm{~T}$. The stimulation intensity was defined in relation to AMT evaluated using the MagPro stimulator. An intensity of $80 \%$ AMT was used. We used the iTBS protocol in which 10 bursts of high frequency stimulation ( 3 pulses at $50 \mathrm{~Hz}$ ) are applied at $5 \mathrm{~Hz}$ every $10 \mathrm{~s}$ for a total of 600 pulses (Huang et al., 2005).

\section{ELF-MFs Exposure}

The system for delivering pulsed ELF-MFs is described in Capone et al. (2009). It consists of a custom-made rectangular, flexible coil kept in place by a Velcro strap and positioned tangential to the inion and to a point $3 \mathrm{~cm}$ above nasion. The magnetic pulse generator (B-01; IGEA, Carpi, Italy) supplied the coil with a single-pulsed signal at $75 \pm 2 \mathrm{~Hz}$, with a pulse duration of $1.3 \mathrm{~ms}$. The peak intensity of the magnetic field was $1.8 \pm 0.2 \mathrm{mT}$. For sham exposure, the coil was applied in the same position but the pulse generator was not turned on. Subjects were blinded for stimulation conditions. ELF-MFs exposure does not give any sensation; for this reason, it is impossible for the subject to distinguish between real from sham exposure. 


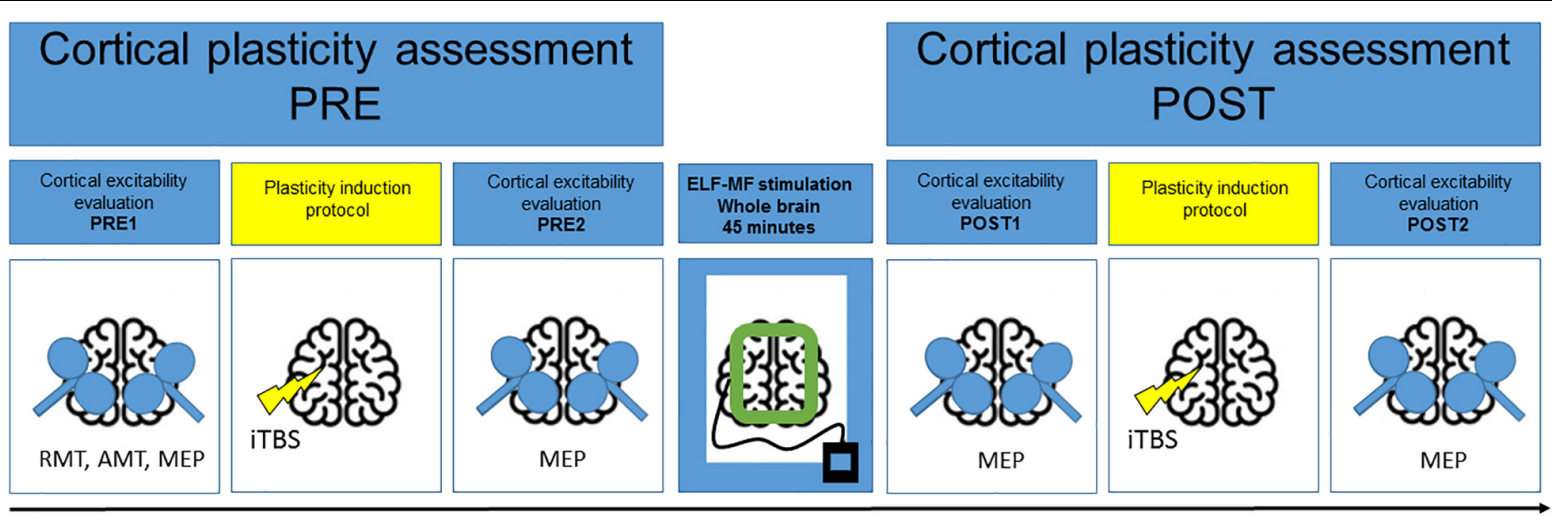

FIGURE 1 | Experimental design. Cortical plasticity was induced by iTBS before and after 45-min ELF-MFs/sham exposure and was estimated by measuring the changes of motor evoked potentials (MEP) amplitude before and after each iTBS.

\section{Statistics}

The sample size was set to be $25 \%$ larger than similar previous studies (Siebner, 2004).

Cortical plasticity was estimated as difference in cortical excitability before and after iTBS. The following measures were therefore computed for both hemispheres:

$$
\begin{aligned}
\Delta \mathrm{MEP}_{\text {pre }} & =\mathrm{MEP}_{\text {pre2 }}-\mathrm{MEP}_{\text {pre } 1} ; \\
\Delta \mathrm{MEP}_{\text {post }} & =\mathrm{MEP}_{\text {post } 2}-\mathrm{MEP}_{\text {post } 1}
\end{aligned}
$$

where pre represents the values obtained before ELF-MFs exposure (pre1 before iTBS and pre 2 after iTBS) and post represents the values recorded after ELF-MFs exposure (post1 before iTBS and post 2 after iTBS). The effect of ELF-MFs on cortical plasticity was tested with a repeated measure ANOVA for each hemisphere, with two factors: stimulation (two levels: Real and Sham) and Time (Pre and Post). The significance level was set to $p<0.05$.

\section{RESULTS}

No adverse events or discomfort sensations were reported. The effects of iTBS and ELF-MFs exposure are illustrated in Figure 2. The database with MEP amplitude raw data is available as Supplementary Material. No significant main factors or interactions were found for both the right and left hemispheres (Figure 3).

\section{DISCUSSION}

This is the first study that has evaluated the effect of ELFMFs on cortical plasticity in the intact human brain. In a previous study, conducted on a different sample using the same technique, we explored the effect of ELF-MFs on TMS measures of cortical excitability (Capone et al., 2009). We found a pronounced increase in intracortical facilitation (ICF) while other parameters such as RMT, AMT, short-interval intracortical

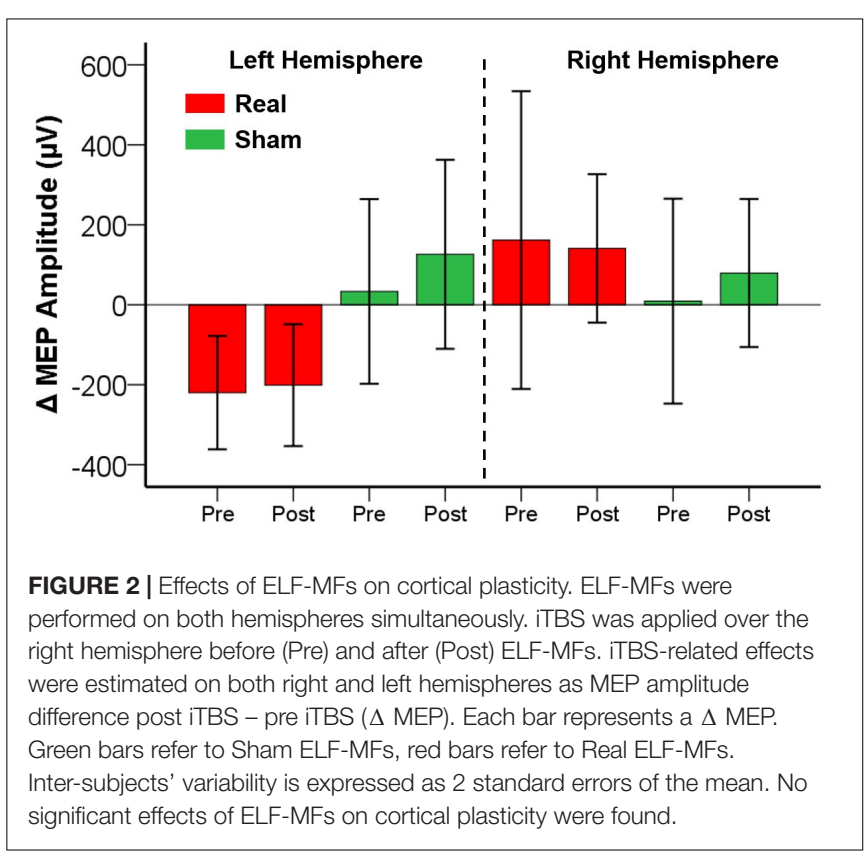

inhibition (SICI) and short-interval afferent inhibition (SAI) remained unchanged. Since ICF mainly reflects excitatory neurotransmission mediated by the NMDA receptors (Di Lazzaro et al., 2008), we hypothesized that ELF-MFs may produce a selective enhancement of glutamatergic activity in human brain.

In the present study we evaluated the effect of ELF-MFs on cortical plasticity, in terms of changes induced by iTBS on TMS measures of cortical excitability. Taken together, our results suggest that although ELF-MFs can influence glutamatergic neurotransmission, they do not affect the propensity of the motor cortex to undergo LTP-like plasticity in vivo. Several in vitro studies have demonstrated that ELF-MFs modulate the activity of different molecules involved in the mechanisms of LTP such as cAMP (Siebner, 2004), glutamate (Hogan and Wieraszko, 2004), and NMDA receptors (Wieraszko et al., 2005). Moreover, they can influence synaptic plasticity toward an 


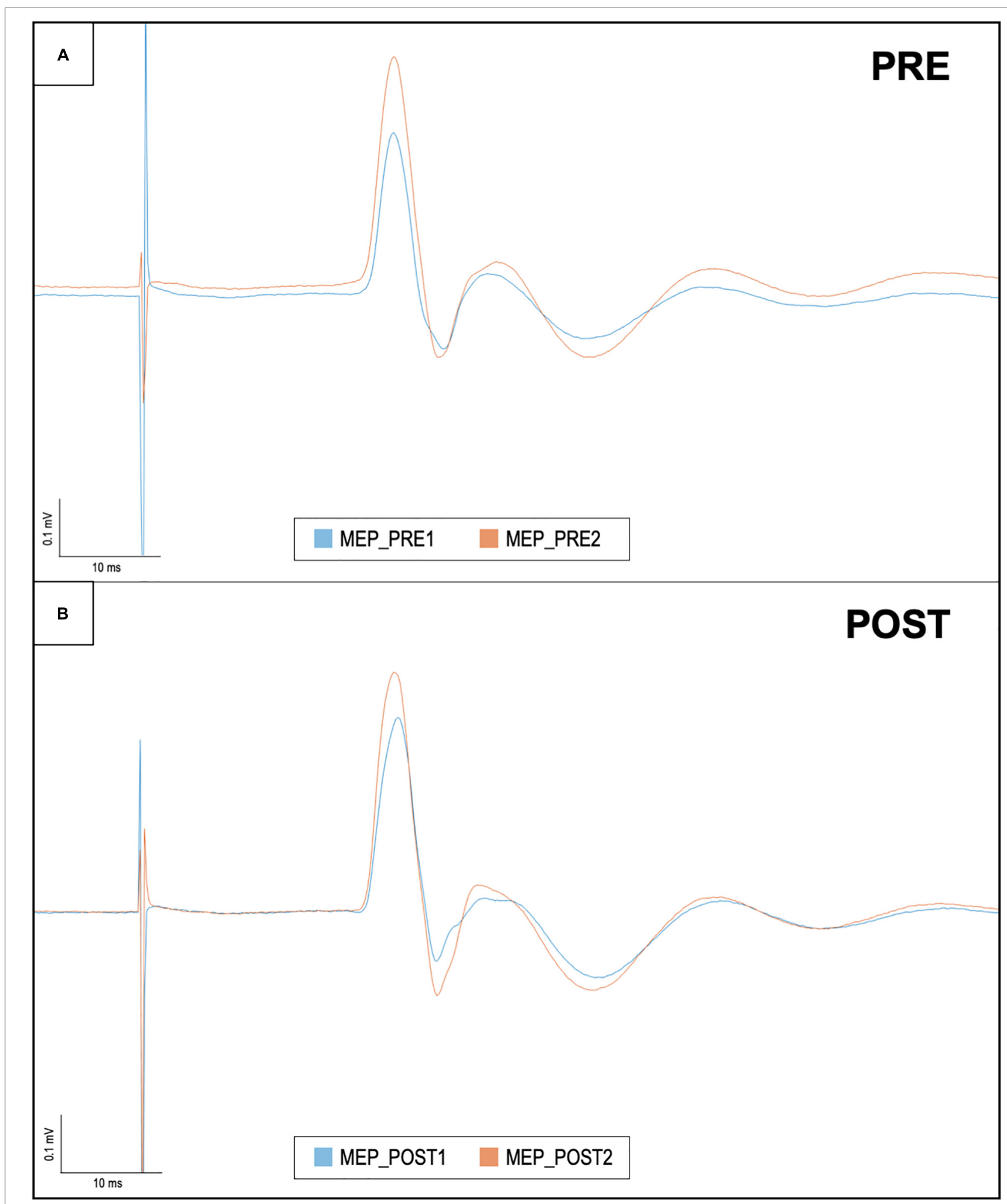

FIGURE 3 | Motor evoked potentials in baseline conditions and after iTBS, before and after ELF-MFs exposure. This figure shows right hemisphere MEP recorded in one representative subject. Each trace is the average of 15 sweeps. (A) The baseline evaluation of cortical plasticity: MEP amplitude was measured before (i.e., MEP_PRE1/blue line) and after iTBS (i.e., MEP_PRE2/orange line). After ELF-MFs exposure, the same evaluation was performed again, as shown in (B). Once again, MEP amplitude was measured before (i.e., MEP_POST1/blue line) and after iTBS (i.e., MEP_POST2/orange line). 
increase (Varró et al., 2009) or a decrease (Balassa et al., 2013) of synaptic strength according to the structural features and neuronal network investigated in the model. Discrepancies between in vivo and in vitro findings could suggest that the effect of ELF-MFs on synaptic plasticity could be counteracted, in vivo, by homeostatic plasticity phaenomena that occur in the intact brain, as already demonstrated for other non-invasive brain stimulation techniques (Müller-Dahlhaus and Ziemann, 2015). This hypothesis is mainly supported by animal studies showing that ELF-MFs exposure has different effects when applied on brain slices as opposed to whole brain (Varró et al., 2009) and by human studies where ELF-MFs modulate glutamatergic neurotransmission without inducing changes in plasticity (Capone et al., 2009).

Other possibilities should be also considered. Since both iTBS and ELF-MFs seem to act on glutamatergic transmission, therefore it is possible that previous exposure to iTBS precludes the development of further plasticity induced by ELF-MFs (ceiling effects/metaplasticity) (Manikonda et al., 2007). Finally, we cannot rule out that the simultaneous excitatory stimulation of both hemisphere by ELF-MFs could produce a null net effect because of an interhemispheric interaction.

Our study has a number of limitations. First, the small sample size of this proof-of-concept study preclude us to draw any definitive conclusions. Second, we explored only the effect of ELF-MFs on motor cortex, while not considering other brain areas (e.g., prefrontal and frontal cortex, somatosensory cortex). Third, the influence of ELF-MFs on cortical plasticity was estimated by measuring the changes in MEP amplitude without considering other important parameters such as input/output curves, RMT, and AMT. Moreover, the number of TMS responses (15) averaged for obtaining MEP amplitude, although in line with previous similar studies (Di Lazzaro et al., 2008, 2011) could be insufficient to capture the effect of ELF-MFs exposure. Finally, we did not perform neuropsychological tests to evaluate the effect of ELF-MFs on cognitive functions related to LTP-like plasticity such as memory or learning processes, but we only relied on neurophysiological measures.

\section{CONCLUSION}

In conclusion, this pilot study did not unveil any significant effect of 45-min whole-brain exposure to ELF-MFs $(75 \mathrm{~Hz}$, $1.8 \mathrm{mT}$ ) on LTP-like plasticity in motor cortex. In light of these results, the effects of ELF-MFs on memory and learning reported by some previous studies could depend on: (a) modulation of

\section{REFERENCES}

Ahmed, Z., and Wieraszko, A. (2008). The mechanism of magnetic field-induced increase of excitability in hippocampal neurons. Brain Res. 1221, 30-40. doi: 10.1016/j.brainres.2008.05.007

Bagheri Hosseinabadi, M., Khanjani, N., Ebrahimi, M. H., Haji, B., and Abdolahfard, M. (2019). The effect of chronic exposure to extremely lowfrequency electromagnetic fields on sleep quality, stress, depression and anxiety. Electromagn. Biol. Med. 38, 96-101. doi: 10.1080/15368378.2018. 1545665 plasticity mechanisms different from those explored by iTBS (Zhu et al., 2015), (b) selective influence on brain regions specifically involved in memory such as hippocampus (not investigated in this study), (c) the differential characteristics of ELF-MFs used in the different studies. Additional studies in larger sample and with different exposure systems (e.g., different noninvasive brain stimulation techniques protocols) are mandatory to confirm our results and to better understand the effect of ELF-MFs on the human brain.

\section{DATA AVAILABILITY STATEMENT}

The raw data supporting the conclusions of this article will be made available by the authors, without undue reservation, to any qualified researcher.

\section{ETHICS STATEMENT}

The studies involving human participants were reviewed and approved by the Campus Bio-Medico Ethical Committee. The patients/participants provided their written informed consent to participate in this study.

\section{AUTHOR CONTRIBUTIONS}

FC and VD designed the study. FM, MR, and GM acquired the data. GP involved in the analysis of data. FC and FM wrote the manuscript. MR, GM, GP, and VD critically revised the manuscript.

\section{FUNDING}

This work was supported by grant from the Italian Ministry of Health (Finalizzata 2013 - CO-2013-02358697).

\section{SUPPLEMENTARY MATERIAL}

The Supplementary Material for this article can be found online at: https://www.frontiersin.org/articles/10.3389/fnhum. 2020.00014/full\#supplementary-material

TABLE S1 | MEP recordings.

Balassa, T., Varró, P., Elek, S., Drozdovszky, O., Szemerszky, R., Világi, I., et al. (2013). Changes in synaptic efficacy in rat brain slices following extremely lowfrequency magnetic field exposure at embryonic and early postnatal age. Int. J. Dev. Neurosci. 31, 724-730. doi: 10.1016/j.ijdevneu.2013.08.004

Bear, M. F., and Malenka, R. C. (1994). Synaptic plasticity: LTP and LTD. Curr. Opin. Neurobiol. 4, 389-399. doi: 10.1016/0959-4388(94)90101-5

Capone, F., Dileone, M., Profice, P., Pilato, F., Musumeci, G., Minicuci, G., et al. (2009). Does exposure to extremely low frequency magnetic fields produce functional changes in human brain? J. Neural Trans. 116, 257-265. doi: 10.1007/ s00702-009-0184-2 
Corbacio, M., Brown, S., Dubois, S., Goulet, D., Prato, F. S., Thomas, A. W., et al. (2011). Human cognitive performance in a $3 \mathrm{mT}$ power-line frequency magnetic field. Bioelectromagnetics 32, 620-633. doi: 10.1002/bem. 20676

Di Lazzaro, V., Capone, F., Apollonio, F., Borea, P. A., Cadossi, R., Fassina, L., et al. (2013). A Consensus panel review of central nervous system effects of the exposure to low-intensity extremely low-frequency magnetic fields. Brain Stimul. 6, 469-476. doi: 10.1016/j.brs.2013.01.004

Di Lazzaro, V., Dileone, M., Pilato, F., Capone, F., Musumeci, G., Ranieri, F., et al. (2011). Modulation of motor cortex neuronal networks by rTMS: comparison of local and remote effects of six different protocols of stimulation. J. Neurophysiol. 105, 2150-2156. doi: 10.1152/jn.00781.2010

Di Lazzaro, V., Pilato, F., Dileone, M., Profice, P., Capone, F., Ranieri, F., et al. (2008). Modulating cortical excitability in acute stroke: a repetitive TMS study. Clin. Neurophysiol. 119, 715-723. doi: 10.1016/j.clinph.2007. 11.049

Hogan, M. V., and Wieraszko, A. (2004). An increase in cAMP concentration in mouse hippocampal slices exposed to low-frequency and pulsed magnetic fields. Neurosci. Lett. 366, 43-47. doi: 10.1016/j.neulet.2004.05.006

Huang, Y. Z., Chen, R. S., Rothwell, J. C., and Wen, H. Y. (2007). The aftereffect of human theta burst stimulation is NMDA receptor dependent. Clin. Neurophysiol. 118, 1028-1032. doi: 10.1016/j.clinph.2007.01.021

Huang, Y.-Z., Edwards, M. J., Rounis, E., Bhatia, K. P., and Rothwell, J. C. (2005). Theta burst stimulation of the human motor cortex. Neuron 45, 201-206.

Jadidi, M., Firoozabadi, S. M., Rashidy-Pour, A., Sajadi, A. A., Sadeghi, H., and Taherian, A. A. (2007). Acute exposure to a $50 \mathrm{~Hz}$ magnetic field impairs consolidation of spatial memory in rats. Neurobiol. Learn. Mem. 88, 387-392. doi: 10.1016/j.nlm.2007.07.010

Komaki, A., Khalili, A., Salehi, I., Shahidi, S., and Sarihi, A. (2014). Effects of exposure to an extremely low frequency electromagnetic field on hippocampal long-term potentiation in rat. Brain Res. 1564, 1-8. doi: 10.1016/j.brainres.2014. 03.041

Liu, X., Zuo, H., Wang, D., Peng, R., Song, T., Wang, S., et al. (2015). Improvement of spatial memory disorder and hippocampal damage by exposure to electromagnetic fields in an Alzheimer's disease rat model. PLoS One 10:e0126963. doi: 10.1371/journal.pone.0126963

Manikonda, P. K., Rajendra, P., Devendranath, D., Gunasekaran, B., Channakeshava, Aradhya, R. S. S., et al. (2007). Influence of extremely low frequency magnetic fields on $\mathrm{Ca} 2+$ signaling and NMDA receptor functions in rat hippocampus. Neurosci. Lett. 413, 145-149. doi: 10.1016/j.neulet.2006.11.048

Müller-Dahlhaus, F., and Ziemann, U. (2015). Metaplasticity in human cortex. Neuroscientist 21, 185-202. doi: 10.1177/1073858414526645

Podd, J., Abbott, J., Kazantzis, N., and Rowland, A. (2002). Brief exposure to a $50 \mathrm{~Hz}, 100 \mu \mathrm{T}$ magnetic field: effects on reaction time, accuracy, and recognition memory. Bioelectromagnetics 23, 189-195. doi: 10.1002/bem. 10004

Siebner, H. R. (2004). Preconditioning of low-frequency repetitive transcranial magnetic stimulation with transcranial direct current stimulation: evidence for homeostatic plasticity in the human motor cortex. J. Neurosci. 24, 3379-3385. doi: 10.1523/jneurosci.5316-03.2004

Varró, P., Szemerszky, R., Bárdos, G., and Világi, I. (2009). Changes in synaptic efficacy and seizure susceptibility in rat brain slices following extremely lowfrequency electromagnetic field exposure. Bioelectromagnetics 30, 631-640. doi: 10.1002/bem.20517

Wieraszko, A., Armani, J., Maqsood, N., Raja, H., and Philip, S. (2005). Modification of the synaptic glutamate turnover in the hippocampal tissue exposed to low-frequency, pulsed magnetic fields. Brain Res. 1052, 232-235. doi: 10.1016/j.brainres.2005.06.034

Zhu, G., Liu, Y., Wang, Y., Bi, X., and Baudry, M. (2015). Different patterns of electrical activity lead to long-term potentiation by activating different intracellular pathways. J. Neurosci. 35, 621-633. doi: 10.1523/jneurosci.219314.2015

Conflict of Interest: The authors declare that the research was conducted in the absence of any commercial or financial relationships that could be construed as a potential conflict of interest.

Copyright (C 2020 Capone, Pellegrino, Motolese, Rossi, Musumeci and Di Lazzaro. This is an open-access article distributed under the terms of the Creative Commons Attribution License (CC BY). The use, distribution or reproduction in other forums is permitted, provided the original author(s) and the copyright owner(s) are credited and that the original publication in this journal is cited, in accordance with accepted academic practice. No use, distribution or reproduction is permitted which does not comply with these terms. 